\title{
MIMO-SAR Imaging Based on Double Frequency Slope Waveform
}

\author{
Xianglin Tan \\ Research Institute of Electronic Science and Technology \\ University of Electronic Science and Technology of \\ China (UESTC) \\ Sichuan, Chengdu, China 611731 \\ txl_uestc@163.com
}

\begin{abstract}
An imaging approach based on the Double Frequency Slope (DFS) waveform in multiple-input and multiple-output (MIMO) synthetic aperture radar (SAR) is presented in this paper. Firstly the DFS waveform's rangeDoppler domain's features after in range direction is analyzed based on principle of stationary phase. Then the phase compensation in the range-Doppler domain is given. Combined with phase characteristic of the multi-antenna, the imaging of MIMO-SAR is achieved. The influence is eliminated because of the double frequency slope, and the sidelobe level in the range direction are reduced by this method. Finally simulation experiments of MIMO-SAR show the correctness and effectiveness of this approach.
\end{abstract}

Keywords-multiple-input multiple-output (MIMO); matching filtered; synthetic aperture radar (SAR); double frequency slope(DFS); radar imaging

\section{INTRODUCTION}

High-resolution and wide swath are two of the most important directions for SAR technology, while the actual achievable resolution and swath for one SAR are subject to the restrictions imposed by several operation factors. Simultaneous high resolution and wide swath cannot be obtained for conventional single-antenna SAR systems. The basic reason is pulse repetition frequency (PRF) which details can be found in [1].

Multiple-input and multiple-output (MIMO) radar has drawing much attention in recent years. Firstly, spatial resolution can be improved due to the multiplied communication channels and additional degrees of freedom. Secondly, MIMO radar can exploit the spatial diversity of target scatters opening the way to a variety of new techniques that can improve radar performance [2-5]. MIMO radar technology combined with the conventional SAR can be a new method to get high resolution and wide swath simultaneously.

Transmitting orthogonal waveforms is one of the most important key in MIMO radar system. In order to eliminate the mutual interference between multiple transmitted waveforms and make full use of the radar echo to improve the azimuth collection of information, the signal should be orthogonal and its auto-correlation function should have low side lobe noise [6-8]. Discrete

\author{
Zhulin Zong \\ Research Institute of Electronic Science and Technology \\ University of Electronic Science and Technology of \\ China (UESTC) \\ Sichuan, Chengdu, China 611731 \\ zongzhulin@uestc.edu.cn
}

frequency-coding waveform (DFCW) [9] and orthogonal frequency division multiplexing (OFDM) [10-11]are two kinds of typical orthogonal waveforms which are widely used in MIMO-SAR. However, the design method of DFCW is complex and the system bandwidth of OFDM is wide, besides both of them are quite sensitive to Doppler shift. Furthermore, auto correlation and cross correlation are relatively poor when the system need to transmit many waveforms. While rang resolution improvement mainly depends on auto correlation main lobe.

Because of its good cross-correlation, auto-correlation and low Doppler sensitivity, a double frequency slope (DFS) waveform is used for MIMO-SAR wide swath sensing in this paper [12]. However due to the different frequency slope of DFS, the phase of the echo signal in the range-Doppler domain is different from linear frequencymodulated, and the traditional imaging algorithms cannot be used directly. So the phase compensation was given based on the DFS waveform's range-Doppler domain's features. And the high resolution SAR image was got finally.

\section{MIMO SIGNAL MODEL}

Double Frequency Slope waveform is made up of two sub-pulses. Each sub-pulse is generalization of linear frequency-modulated (LFM) waveform and has equal time-duration. In LFM waveform, frequency changes at a same frequency slope, whereas in DFS waveform, there are two different frequency slopes as depicted in Fig.1.

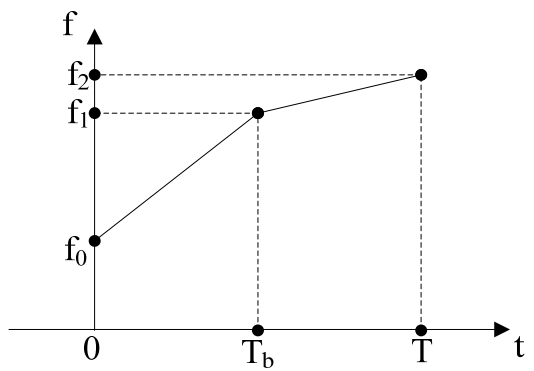

Figure 1. Time-Frequency relationship of DFS signal

It is assumed that $M$ radars transmit different DFS waveforms and each DFS waveforms share the same 
carrier frequency, bandwidth and time-duration, $\mathrm{T}$ is pulse length, $T_{b}=T / 2$ is the sub-pulse length. $k_{\text {in }}$ $(i=1,2, \cdots, M, n=1,2)$ is two chirp rate of DFS waveform. Then the transmitting signal $S_{i}(t)(i=1,2, \cdots, M)$ can be described as follow:

$$
\begin{aligned}
& S_{m}(t)=\sum_{i=1}^{2} \exp \left\{j \cdot 2 \pi f_{0} t+j \cdot \pi \sum_{k=0}^{i-1} k_{m k} T_{b}^{2}+\right. \\
& j \cdot 2 \pi \cdot\left(\sum_{k=0}^{n-1} k_{m k}\right) \cdot T_{b} \cdot\left[t-(\mathrm{i}-1) T_{b}\right]+ \\
& \left.j \cdot \pi k_{m i}\left[t-(i-1) T_{b}\right]^{2}\right\} \cdot \operatorname{rect}\left[\left(t-\left(i-\frac{1}{2}\right) T_{b}\right) / T_{b}\right]
\end{aligned}
$$

Auto-correlation, cross-correlation and Doppler sensitivity of DFS waveform have been investigate in the [12]. By setting the waveform parameters (time-duration, bandwidth, step of frequency slope), the orthogonal performance can be improved to meet requirement of MIMO-SAR image, and the side lobes can be reduced to obtain high resolution.

\section{THE MODEL OF MIMO-SAR SYSTEM}

Considering the number $\mathrm{M}$ of antennas in the linear aperture array which located along the azimuth direction uniformly can transmit and receive orthogonal waveform, and the aperture synthesis is employed by the platform moving which is called MIMO-SAR radar system [13]. The principle of equivalent phase center is usually used in the MIMO radar system, and then each phase center can be equivalent to one single-input and single output SAR. Equivalent phase centers show in Fig.2 (two transmitting antennas and receiving antennas). Hollow squares and solid squares represent no overlapping data and overlapping data respectively.

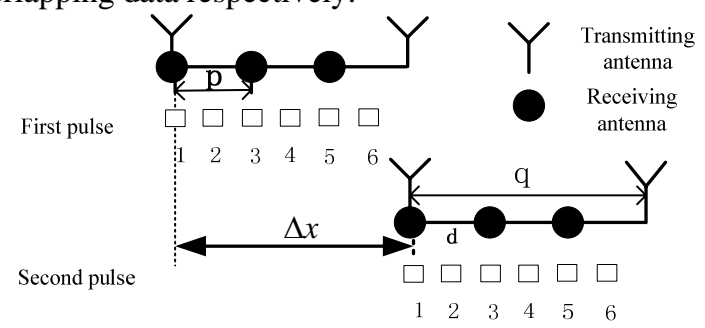

Figure 2. Equivalent phase center

Assumed $\Delta x$ is movement distance of the radar platform during one repetition interval. The range between adjacent transmitting antennas and adjacent receiving antennas is $q, p$. As show in the Fig.2, uniform distribution data can be got when $\Delta x, p$ and $q$ satisfy Equation (2).

$$
q=3 p=\Delta x
$$

However, the equivalent phase center will introduce errors which can be multiplied by following compensation function as Equation (3) [12].

$$
H_{\text {equ }}(m, n)=\exp \left(-j 2 \pi l_{m, n} / \lambda R_{s}\right)
$$

Where $l_{m, n}$ is the distance between transmitting antennas $\mathrm{m}$ and receiving antennas, $\lambda$ is the wavelengt and $R_{\mathrm{s}}$ is the vertical distance from the center of the scene to the flight path. After the errors compensated in the time domain, six times the effective single-channel data can be obtain during a pulse repetition in azimuth direction. Thus the pulse repetition frequency can be reduced to original 1/6. So the contradiction between low pulse repetition frequency and high resolution can be solved.

\section{MAtChed Filtering And Phase Compensation METHOD}

Due to two different frequency slopes of DFS waveform, matching function of LFM cannot be applied directly to DFS in the RD imaging algorithm. Through the analysis of the echo waveform, rang matching function is given to the DFS. According to the characteristics of rang Doppler domain, phase compensation method is deduced.

Assumed $\mathrm{M}$ antennas transmit different DFS signal whose waveforms are the same as $S_{i}(t)(i=1,2, \cdots \mathrm{M})$ in the Equation 1. Those waveforms mixed together after reflected from the target which can be received by each array in a synthetic aperture time. Because of the orthogonality of the DFS waveform, each of the transmitted waveform can be separated from echo through Correlation processing. This process can be performed by convolution with a matching function. Then the signal received by the $\mathrm{m}^{\text {th }}$ receiving antenna from the $\mathrm{nth}$ transmitting antenna can be expressed as:

$$
\begin{aligned}
& S_{m n}\left(t ; \mathrm{R}_{\mathrm{m}, \mathrm{n}}\right)=\sum_{i=1}^{2} \exp \left\{j \cdot 2 \pi f_{0}\left(t-\tau_{m n}\right)+j \cdot \pi \sum_{k=0}^{i-1} k_{m k} T_{b}^{2}+\right. \\
& j \cdot 2 \pi \cdot\left(\sum_{k=0}^{n-1} k_{m k}\right) \cdot T_{b} \cdot\left[t-(\mathrm{i}-1) T_{b}-\tau_{m n}\right]+ \\
& \left.j \cdot \pi k_{m i}\left[t-(i-1) T_{b}-\tau_{m n}\right]^{2}\right\} \cdot \operatorname{rect}\left[\frac{t-\left(i-\frac{1}{2}\right) T_{b}-\tau_{m n}}{T_{b}}\right]
\end{aligned}
$$

Where $\tau_{m n}=\mathrm{R}_{m n} / c$ is the time delay, c is the speed and $\mathrm{R}_{m n}(\eta ; r)$ is the distance from equivalent phase center of the mth transmitting antenna and the nth receiving antenna, $\eta$ is the slow time in azimuth direction.

According to the basic process of $\mathrm{RD}$ algorithm, Equation (4) should be transformed to range frequency domain firstly. Two different frequency slopes of DFS have different and equal time and each sub-pulse satisfies principle of stationary phase. Based on the accumulation 
of Fast Fourier Transform (FFT), Two sub-pulse signal in rang Doppler domain can be accumulated. Then we get the FFT of $S_{m n}\left(t ; R_{m, n}\right)$

$$
\begin{aligned}
& S s_{m n}(f: r)=r e c t\left[\frac{\frac{f}{k_{m 1}}-\frac{T_{b}}{2}}{T_{b}}\right] \exp \left(-j \pi \frac{f^{2}}{k_{m 1}}-2 j \pi\left(f_{0}+f\right) \tau_{m n}\right)+ \\
& \operatorname{rect}\left[\frac{\frac{f}{k_{m 2}}-\frac{T_{b}}{2}-\frac{k_{m 1}}{k_{m 2}} T_{b}}{T_{b}}\right] \exp \left(-j \pi \frac{f^{2}}{k_{m 2}}-2 j \pi\left(f_{0}+f\right) \tau_{m n}\right) . \\
& \exp \left\{2 j \pi\left(\frac{k_{m 1}}{k_{m 2}}-1\right) T_{b} f+j \pi k_{m 1} T_{b}^{2}-j \pi \frac{k_{m 1}^{2}}{k_{m 2}} T_{b}^{2}\right\} .
\end{aligned}
$$

As show in Fig.1, DFS waveform share the time of the positive semi axis, so the matched filtering function in the rang directing can be described as $S_{r}(t)=S_{i}\left(2 T_{b}-t\right)$ :

$$
\begin{gathered}
S_{r}(t)=\operatorname{rect}\left[\frac{\frac{3 T_{b}}{2}-t}{T_{b}}\right] \exp \left\{-j \pi k_{p 1}\left(2 T_{b}-t\right)^{2}\right\}+ \\
\operatorname{rect}\left[\frac{\frac{T_{b}}{2}-t}{T_{b}}\right] \exp \left\{-j \pi k_{p 2}\left(T_{b}-t\right)^{2}\right\} . \\
\exp \left\{j \pi k_{p 1} T_{b}^{2}-j 2 \pi k_{p 1} T_{b}\left(2 T_{b}-t\right)\right\}
\end{gathered}
$$

Based on principle of stationary phase as well, we take the range Fourier transform of $S_{r}(t)$ to get the $S s_{r}(f, \eta ; r)$ :

$$
\begin{gathered}
\left.{S s_{r}}_{r}(f, \eta ; r)=r e c t\left[\left(\frac{f}{k_{m 1}}-\frac{T_{b}}{2}\right) / T_{b}\right] \exp \left(\frac{\pi f^{2}}{k_{m 1}}-2 \pi j \cdot 2 T_{b} f\right)\right)+ \\
\operatorname{rect}\left[\left(\frac{f}{k_{m 1}}-\frac{T_{b}}{2}-\frac{k_{m 1}}{k_{m 2}} T_{b}\right) / T_{b}\right] \exp \left(\frac{\pi f^{2}}{k_{m 2}}\right) . \\
\quad \exp \left(j \pi k_{m 1}\left(\frac{k_{m 1}}{k_{m 2}}-1\right) T_{b}^{2}-2 \pi j\left(\frac{k_{m 1}}{k_{m 2}}+1\right) T_{b} f\right)
\end{gathered}
$$

From Equation (6) and (7), we can see that each subpulse of $S_{m n}\left(t ; R_{m n}\right)$ and $S s_{r}\left(f ; R_{m n}\right)$ share the same frequency. So only $S_{m n}\left(t ; R_{m n}\right)$ and $S s_{r}\left(f ; R_{m n}\right)$ two subpulses multiplied respectively, then we can get

$$
\begin{aligned}
S^{\prime}\left(f ; R_{m n}\right) & =\operatorname{rect}\left[\frac{\frac{f}{k_{m 1}+k_{m 2}}-\frac{T_{b}}{2}}{T_{b}}\right] \exp \left(-2 \pi j\left(f_{0}+f\right) \tau_{m n}\right) . \\
& \exp \left(-2 \pi j \cdot 2 f T_{b}\right)
\end{aligned}
$$

Contrast of Equation (8) and LFM after matching filter waveform, phase compensation function can be described as:

$$
H(f)=\operatorname{rect}\left[\left(\frac{f}{k_{m 1}+k_{m 2}}-\frac{T_{b}}{2}\right) / T_{b}\right] \exp \left(2 \pi j \cdot 2 f T_{b}\right)
$$

Equation (8) is to be multiplied by Equation (9), We get the data in range Doppler domain after the matching filter finally

$$
\begin{aligned}
S(f, \eta ; r) & =S^{\prime}(f, \eta ; r) \cdot H(f) \\
& =\operatorname{rect}\left[\left(\frac{f}{k_{1}+k_{2}}-\frac{T_{b}}{2}\right) / T_{b}\right] \exp \left(-2 \pi j\left(f_{0}+f\right) \tau\right)
\end{aligned}
$$

From Equation (10) we can see that the spectrum of DFS waveform after phase compensation is the same as LFM in conventional SAR system. So the subsequent signal processing of DFS in the azimuth direction can be taken as LFM waveform in regular SAR.

\section{Simulation Result}

Strip-map MIMO-SAR data from a point target are simulated in this section using the parameters listed in Table 1.

TABLE I. MAIN SIMULATION PARAMETERS

\begin{tabular}{|c|c|c|}
\hline Parameters & Values & Units \\
\hline Carrier frequency & 10 & {$[\mathrm{GHz}]$} \\
\hline Platform velocity & 300 & {$[\mathrm{~m} / \mathrm{s}]$} \\
\hline Platform altitude & 6000 & {$[\mathrm{~m}]$} \\
\hline Range resolution & 1.5 & {$[\mathrm{~m}]$} \\
\hline Azimuth Resolution & 1.5 & {$[\mathrm{~m}]$} \\
\hline $\begin{array}{c}\text { Azimuth aperture of each } \\
\text { antenna }\end{array}$ & 3 & {$[\mathrm{~m}]$} \\
\hline Bandwidth & 100 & {$[\mathrm{M}]$} \\
\hline PRF & 50 & {$[\mathrm{~Hz}]$} \\
\hline
\end{tabular}

The other parameters of DFS waveform design can be found in [13]. The pulse length $T_{p}=800 \cdot(M-1)^{2} \cdot M / B$, $\mathrm{M}$ is the number of the radar and $\mathrm{B}$ is the bandwidth. The average frequency modulation slope of DFS is $k=B / T$, so $k_{m 1}=k+m \cdot \Delta k$ and $k_{m 2}=k-m \cdot \Delta k(m=1,2,3)$ are two different frequency modulation slopes of each DFS. The simulation image as shown below: 


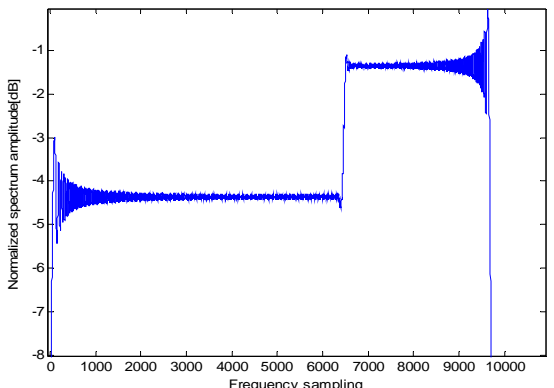

Figure 3. Spectrum after match matched filtering

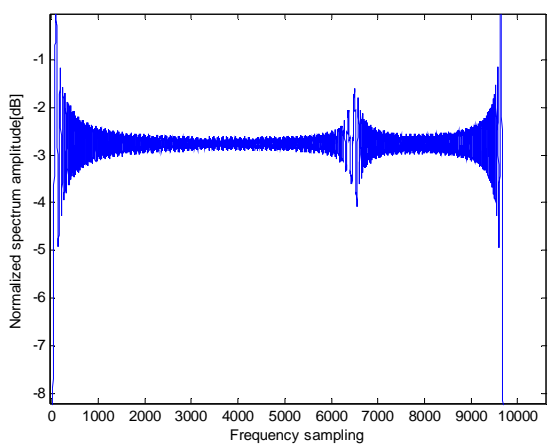

Figure 4. Spectrum after phase compensation

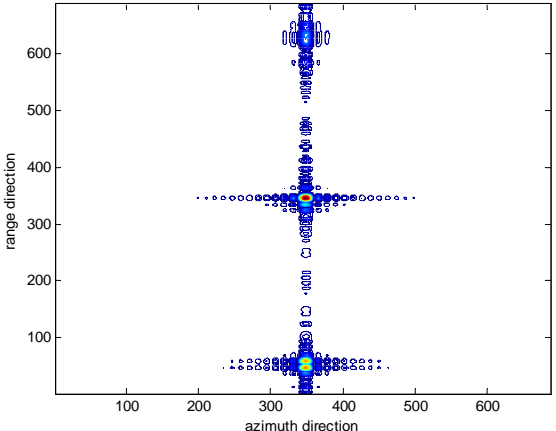

Figure 5. Imaging simulation result of SAR

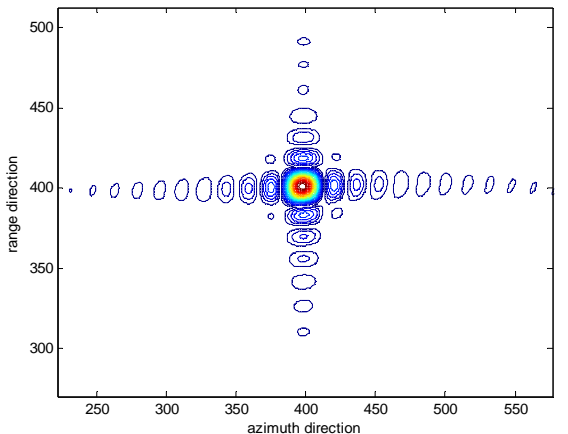

Figure 6. Imaging simulation result of MIMO-SAR
Contrasting Fig.3 and Fig.4, the spectrum of DFS waveform after phase compensation is the same as LFM and the influence brought by two frequency different slopes is eliminated. As show in Fig.5, the image is ambiguities in the azimuth direction when the PRF below the Doppler bandwidth in conventional SAR, however the image is focused well in the MIMO-SAR radar system with the equal PRF which can be seem from Fig.6.

\section{CONCLUSION}

The DSF waveform involves two different frequency slopes with good cross-correlation and low Doppler sensitivity. This paper deduces the phase compensation method of DFS in the RD algorithm, and gets the MIMOSAR image which solves the contradiction between highresolution and wide swath.

\section{REFERENCES}

[1] Currie and M. A. Brown, "Wide-swath SAR,” Proc. Inst. Elect. Eng.-F, Radar Signal Process. , vol. 139, no. 2, pp. 122-135, Apr. 1992

[2] Peng F X, Li H W, Cai B, et al.Ultra-High Resolution Wide Swath Imaging with Spaceborne MIMO-SAR[J]. Radar Science and Technology, 2011,09(05):409-429.

[3] Gu W K, Wang D W, MA X Y. Algorithm for Large Scene Imaging of Airborne MIMO Radar. Journal of Air Force Radar Academy, 2011,25(02):79-86.

[4] Klare Jens, Ender Joachim H. G. "System architectures and algorithms for radar imaging by MIMO-SAR". Radar Conference, 2009 IEEE, pp.1097-5659.

[5] Wang Wenqin, Cai Jingye. MIMO SAR using Chirp Diverse Waveform for Wide-Swath Remote Sensing[J]. IEEE Transactions on aerospace and electronic systems. 2011, 48(4):3171:3184.

[6] Robey F C, Coutts S, Weille D and et al. MIMO radar theory and experimental Conference Record of the Thirty-Eighth Asilomar conference on Signals, Systems and Computer, 2004, vol.1:300304.

[7] Li,J. and Stoica, P. MIMO Radar Signal Processing Hoboken, NJ:Wiley,2009.

[8] Kriger G,GebertN,Moreira A.Multidimensioanl waveform encoding: a new digital beamforming technique for synthetic aperture radar remote sensing[J]. IEEE Transactions on Geoscience and Remote Sensing, 2008,46(1):31-46.

[9] Fishler E, Haimovich A, Blum R, Chizhik D, Cimini L, Valenzuela R. MIMO Radar: an Idea Whose Time Has Come. In Proc IEEE radadr Conference, 2004, vol.1:71-78.

[10] Wang Wenqin. Space-Time Coding MIMO-OFDM SAR for HighResolution Imaging. IEEE Transactions on Geoscience and Remote Sensing. 2011,49(8):3094-3104.

[11] F Cheng, Z. S. Hu, H.M.Liu, and J. Li, "The parameter setting problem of signal OFDM-LFM for MIMO radar”, in Proc. Int. Commun., Circuits syst. Conf., Fujian, China, May 2008:876-880.

[12] Zong Z L.Study on Waveform design and Imaging Technology for Formation-Flying Satellites SAR. Chengdu, University of Electronic Science and Technology of China. 2012

[13] Wang Libao, Jia Xu, Kan Huangfu, Peng Yingning. “Analysis and Compensation of Equivalent Phase Center Error in MIMO-SAR”. Acta Electronica Sinica, 2009, 37(12), pp. 2688-2693 